\title{
The effect of hiatus hernia on gastro-oesophageal junction pressure
}

\author{
P J Kahrilas, S Lin, J Chen, M Manka
}

\begin{abstract}
Background-Hiatus hernia and lower oesophageal sphincter hypotension are often viewed as opposing hypotheses for gastro-oesophageal junction incompetence.

Aims-To examine the interaction between hiatus hernia and lower oesophageal sphincter hypotension.

Methods-In seven normal subjects and seven patients with hiatus hernia, the squamocolumnar junction and intragastric margin of the gastro-oesophageal junction were marked with endoscopically placed clips. Axial and radial characteristics of the gastro-oesophageal junction high pressure zone were mapped relative to the hiatus and clips during concurrent fluoroscopy and manometry. Responses to inspiration and abdominal compression were also analysed.

Results-In normal individuals the squamocolumnar junction was $0.5 \mathrm{~cm}$ below the hiatus and the gastro-oesophageal junction high pressure zone extended 1.1 cm distal to that. In those with hiatus hernia, the gastro-oesophageal junction high pressure zone had two discrete segments, one proximal to the squamocolumnar junction and one distal, attributable to the extrinsic compression within the hiatal canal. Inspiration and abdominal compression mainly augmented the distal one. Simulation of hernia reduction by algebraically summing the proximal segment pressures with the hiatal canal pressures restored normal maximal pressure, radial asymmetry, and dynamic responses of the gastro-oesophageal junction.
\end{abstract}

Conclusions-Hiatus hernia reduces lower oesophageal sphincter pressure and alters its dynamic responsiveness by spatially separating pressure components derived from the intrinsic lower oesophageal sphincter and the extrinsic compression of the oesophagus within the hiatal canal.

(Gut 1999;44:476-482)

Keywords: hiatus hernia; lower oesophageal sphincter; reflux disease; manometry

Investigations into the mechanisms of gastrooesophageal reflux have identified transient lower oesophageal sphincter (LOS) relaxation as a dominant mechanism, but also observed that strain induced reflux or free reflux could occur during periods of LOS hypotonicity. ${ }^{12}$ These studies also emphasised the variability of each individual's LOS pressure over time during prolonged recordings and the relatively poor sensitivity of this measurement for the detection of reflux disease. None the less, LOS hypotonicity does become increasingly common with increasing severity of oesophagitis, ${ }^{34}$ and multivariate analysis does suggest LOS hypotonicity to be an independent risk factor for both the presence and grade of oesophagitis. ${ }^{5}$ From a mechanistic viewpoint, factors which diminish the LOS pressure predispose to strain induced or free reflux and these mechanisms are more dominant with increasing grades of oesophagitis. ${ }^{6}$ Manometric data also suggest that strain induced reflux is more common in the setting of hiatus hernia. ${ }^{7}$ Another association that is difficult to ignore is that between oesophagitis and hiatus hernia. Endoscopic and radiographic studies have found that $50-94 \%$ of patients with reflux disease have hiatus hernia, whereas the prevalence of hernias in control subjects was substantially less within each study. ${ }^{8-10}$ Furthermore, stepwise regression analysis of the susceptibility to reflux by the strain mechanism identified increasing size of hiatus hernia, diminishing values of LOS pressure, and an interaction between hernia size and LOS pressure as significant independent risks. ${ }^{11}$ These observations suggest that hiatus hernia may hinder LOS function, presumably by modifying the diaphragmatic contribution to gastro-oesophageal junction competence. ${ }^{12} 13$ With that in mind, the aim of this study was to evaluate the effect of anatomically well defined hiatus hernia on manometric characteristics of the gastro-oesophageal junction pressure profile.

\section{Methods}

Endoscopic, fluoroscopic, and manometric landmarks of the gastro-oesophageal junction of patients with hiatus hernia and volunteer subjects were compared to examine the influence of hiatus hernia on the intraluminal pressure profile. The protocol was approved by the Northwestern University Institutional Review Board and informed consent was obtained from participants.

SUBJECT GROUPS

Subjects for this investigation were a group of normal volunteers and patients with symptomatic reflux disease categorised as having hiatus hernia and without Barrett's epithelium

Abbreviations used in this paper: LOS, lower oesophageal sphincter; SCJ, squamocolumnar junction; HPZ, high pressure zone; GOJ, gastro-oesophageal junction; EMG, electromyography. 


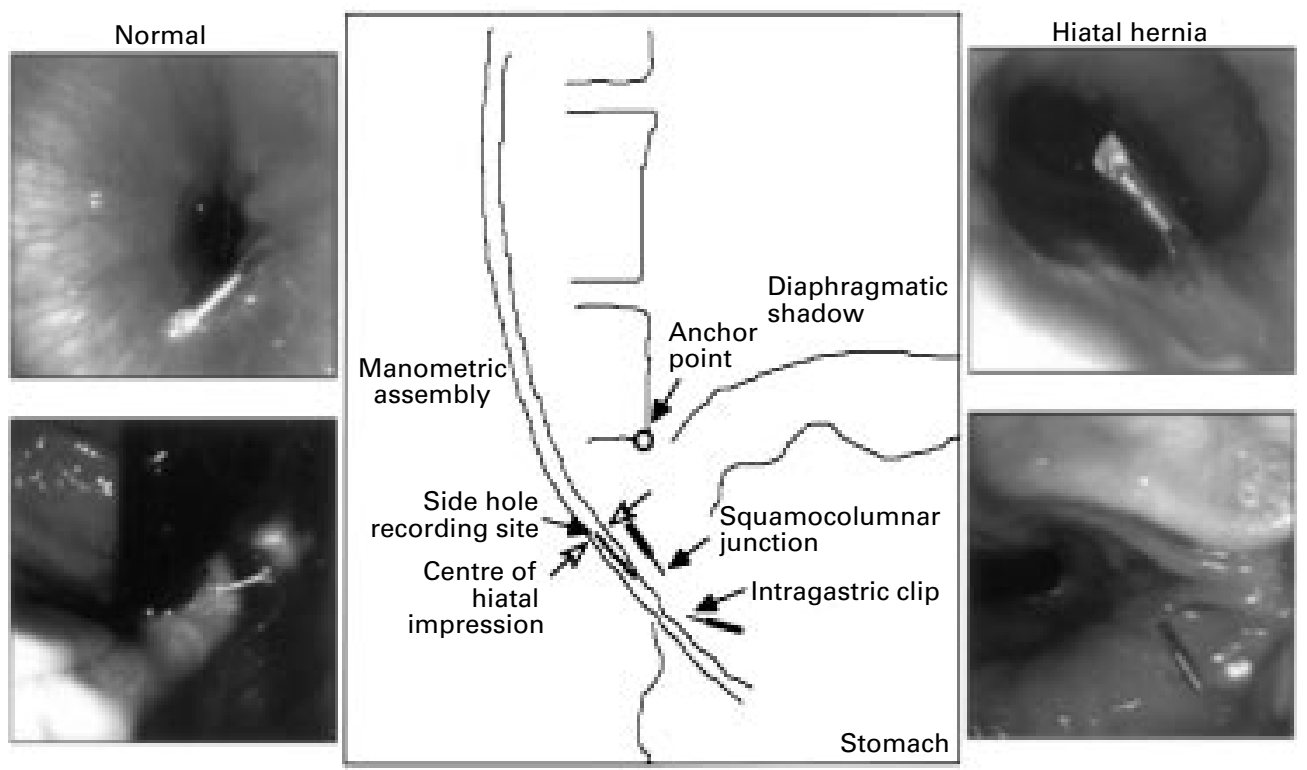

Figure 1 Positioning of mucosal clips in normal subjects and patients with hiatus hernia. The central panel is a tracing from a fluoroscopic image of a normal subject illustrating the position of the mucosal clips relative to fluoroscopic landmarks. The opposing open arrows indicate the position of the centre of the hiatal canal as determined by the impression into the barium column during a barium swallow.

on the basis of prior, clinically indicated, endoscopic evaluation. Of a total of nine volunteers and nine patients evaluated, seven in each group (four men, three women) satisfied our criteria for inclusion after endoscopic and fluoroscopic assessment of the position of the squamocolumnar junction relative to the diaphragm (see below). The mean age of the seven controls was 38 (6) years and that of the hernia patients was 44 (5) years; all of the hernia patients had reflux disease that was in endoscopic and symptomatic remission as a result of maintenance treatment with a proton pump inhibitor $(n=5)$ or a histamine $\mathrm{H}_{2}$ receptor antagonist $(n=2)$.

\section{ENDOSCOPIC LANDMARKS OF THE} GASTRO-OESOPHAGEAL JUNCTION MARKED BY MUCOSAL CLIPPING

Subjects fasted overnight before undergoing an oesophagoscopy under sedation with intravenous midazolam and meperidine. During this procedure, two $11 \mathrm{~mm}$ stainless steel clips were attached to the oesophageal and gastric mucosa using an endoscopic clip fixing device (HX-3L, Olympus America Inc., Lake Success, New York, USA). The proximal clip was attached at the squamocolumnar junction and the distal clip was attached at the greater curvature side of the entryway to the stomach as viewed in retroflexion. If the squamocolumnar junction was irregular the clip was affixed at a median location between its proximal and distal extremes. The anatomical correlate of the intragastric clip was distinct for the two groups; in the normals this correlated with the intragastric flap valve as defined by Hill et $a l,{ }^{14}$ whereas in the hernia subjects the intragastric clip marked the lower margin of the diaphragmatic hiatus (fig 1). Following completion of the clipping procedure, subjects were allowed to recover from sedation for at least one hour before proceeding with the manometric and fluoroscopic studies. The oesophagus was again imaged fluoroscopically one month after the study, and all residual clips were removed endoscopically.

MANOMETRIC AND FLUOROSCOPIC ASSESSMENT OF THE GASTRO-OESOPHAGEAL JUNCTION Prior to manometric study, subjects were imaged fluoroscopically in a supine posture to ascertain their suitability for inclusion in the normal or hiatus hernia groups. Two $5 \mathrm{ml}$ dilute liquid barium swallows (Liquid E-Z, E-Z-EM Inc., Westbury, New York, USA) were imaged to determine the end expiratory position of the diaphragmatic impression on the oesophagus or stomach. Swallows imaged fluoroscopically were recorded with a videotape recorder (Sony VO 9800). Subjects were
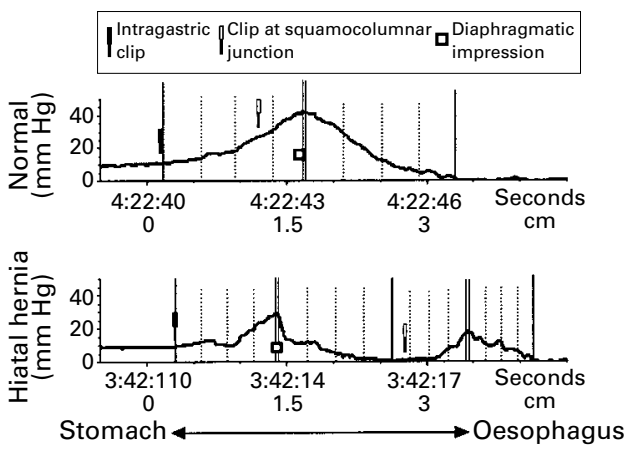

Figure 2 Examples of manometric pull through tracings with single (top) and double (bottom) peak axial pressure profiles. The median axial position and the pressure of the maximum (double vertical lines), minimum (single vertical lines), and three equispaced intermediate positions (vertical dotted lines) of each upward and downward slope were used to summarise group data. The open clips show the position of the squamocolumnar junction, the closed clips indicate the position of the intragastric clip, and the open squares indicate the centre of the hiatal canal. 
Table 1 Gastro-oesophageal junction high pressure zone (HPZ) characteristics of normal subjects and patients with hiatus hernia

\begin{tabular}{|c|c|c|c|}
\hline & \multirow[b]{2}{*}{ Normal subjects } & \multicolumn{2}{|l|}{ Hiatus hernia } \\
\hline & & HPZ 1 (proximal) & HPZ 2 (hiatal) \\
\hline Maximal pressure $(\mathrm{mm} \mathrm{Hg})$ & $28(1.2)$ & $17(1.0)^{\star}$ & $13(2.0)^{\star}$ \\
\hline \multicolumn{4}{|l|}{ Maximal pressure (mm Hg, referenced } \\
\hline to gastric pressure) & $17(1.0)$ & $7(0.2)^{\star}$ & $3(0.2)^{\star}$ \\
\hline Percentage radial asymmetry & $34(2)$ & $3(1)^{\star}$ & $52(2)^{\star}$ \\
\hline Length of high pressure zone $(\mathrm{cm})$ & $4.0(0.2)$ & $2.8(0.2)^{\star}$ & $2.7(0.3)^{\star}$ \\
\hline
\end{tabular}

All values are mean (SEM)

${ }^{\star} \mathrm{p}<0.05$ versus normal.

Normal
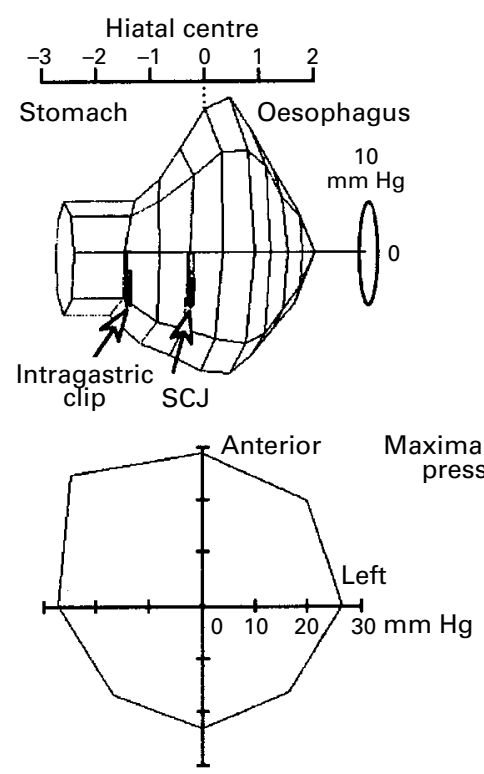

Figure 3 Axial (top) and radial (bottom) pressure topography of the gastro-oesophageal junction of normal subjects and patients with hiatus hernia. The radial pressure profiles at the peak of each high pressure zone are diagrammed underneath. Position zero on the axial scale is the midpoint of the diaphragmatic hiatus. The wireframe representations are rotated such that the right anterior pressure is at the top and left posterior pressure is at the bottom, thereby accentuating the radial pressure asymmetry. The proximal clip indicates the median position of the squamocolumnar junction (SCF) and the distal clip marks the median position of the intragastric aspect to the gastro-oesophageal junction as imaged endoscopically. All values of length and pressure are the medians of the subject groups.

then imaged at a time that they had not swallowed for at least 30 seconds to ascertain the position of the mucosal attachment of the squamocolumnar junction clip relative to the position of the hiatal impression that had been ascertained during the barium swallows. Measurements were made from digitised images using the vertebrae as spatial references, the length of the clips to correct for magnification, and image analysis software. ${ }^{15}$ To qualify as normal, the squamocolumnar junction clip needed to be beneath the diaphragmatic hiatus

Table 2 Changes in pressure, length, and position of the gastro-oesophageal junction (GOF) high pressure zone (HPZ) caused by abdominal compression

\begin{tabular}{llll}
\hline & \multicolumn{3}{l}{ Hiatus hernia } \\
\cline { 3 - 4 } & $\begin{array}{l}\text { Normal } \\
\text { subjects }\end{array}$ & $\begin{array}{l}\text { HPZ 1 } \\
\text { (proximal) }\end{array}$ & $\begin{array}{l}\text { HPZ } 2 \\
\text { (hiatal) }\end{array}$ \\
\hline Intragastric pressure change (mm Hg) & $7(2.0)$ & - & $6(2.1)$ \\
Increase in maximal GOJ pressure (mm Hg) & $13(1.2)$ & $4(0.4)^{\star}$ & $13(1.5)$ \\
Length change of high pressure zone (cm) & $-0.1(0.1)$ & $-0.2(0.1)$ & 0 \\
Movement of locus of maximal pressure (cm) & $0.5(0.1)$ & $0.6(0.1)$ & $0.4(0.1)$ \\
\hline
\end{tabular}

Changes in patients with hiatus hernia were measured both within the proximal high pressure zone (HPZ 1) and the hiatal canal (HPZ 2). All values are mean (SEM).

${ }^{\star} \mathrm{p}<0.05$ versus normal. while our requirement for hiatus hernia was that the squamocolumnar junction clip be at least $1 \mathrm{~cm}$ proximal to the hiatus. Application of these criteria resulted in exclusion of two normal subjects whose squamocolumnar junction was slightly above the diaphragm and two patients with hiatus hernia whose squamocolumnar junction was less than $1 \mathrm{~cm}$ above the diaphragm.

Subjects were then studied with concurrent fluoroscopy and manometry. A silicone rubber manometric assembly with eight side hole recording sites situated at the same axial level but arranged at $45^{\circ}$ increments radially was used (Dentsleeve Pty, 77C Gibson St, Bowden, South Australia). Asymmetric radio-opaque markings on the manometric assembly permitted fluoroscopic determination of axial position and radial orientation of the side hole sites. Each catheter lumen was perfused by a low compliance perfusion pump at $0.3 \mathrm{ml} / \mathrm{min}$ (Dentsleeve Mark II, 16 channel model), connected to a computer polygraph set at a sampling frequency of $40 \mathrm{~Hz}$ (Neomedix Systems Pty Ltd, Warriewood, NSW, Australia), and processed utilising Gastromac software (Neomedix). Response characteristics of each manometric channel exceeded $200 \mathrm{~mm}$ $\mathrm{Hg} / \mathrm{s}$. Data for determination of the gastrooesophageal junction pressure topography were obtained during fluoroscopically imaged pull throughs of the gastro-oesophageal junction at $0.5 \mathrm{~cm} / \mathrm{s}$ using a mechanical catheter pulling device (Medtronic Synectics, 3850 Victoria Street North, Mail Stop V215, Shoreview, Minnesota, USA). Manometric tracings and fluoroscopic images were synchronised using a video timer (model VC 436, Thalner Electronics Laboratories, Ann Arbor, Michigan, USA) that encoded time in hundredths of a second on each video frame and sent a $1 \mathrm{~V}-10$ ms pulse to an instrumentation channel of the polygraph at whole second intervals. Pull throughs were done during suspended respiration in three conditions: end expiration; strong respiratory effort with a closed glottis (Müller manoeuvre); and end expiration with an abdominal binder inflated to $100 \mathrm{~mm} \mathrm{Hg}$.

\section{DATA ANALYSIS}

The recording from each manometric lumen of each pull through was analysed to relate the pressure signal with the squamocolumnar junction, the intragastric clip, and the fluoroscopic position of the hiatal indentation as determined during the initial barium swallows. This was accomplished by digitising selected images and relating the position of the asymmetric radio-opaque markers on the manometric assembly to the mucosal attachment points of the clips at each time point of the pull through. Axial position zero was defined as the midpoint of the hiatal canal; all pressures were referenced to atmospheric. Pressure tracings were then characterised as having a single or double peak (fig 2). Each slope of each peak was further characterised by the axial location and pressure value of the peak, nadir, and three equispaced intermediate points. Thus, pressure values were obtained for 

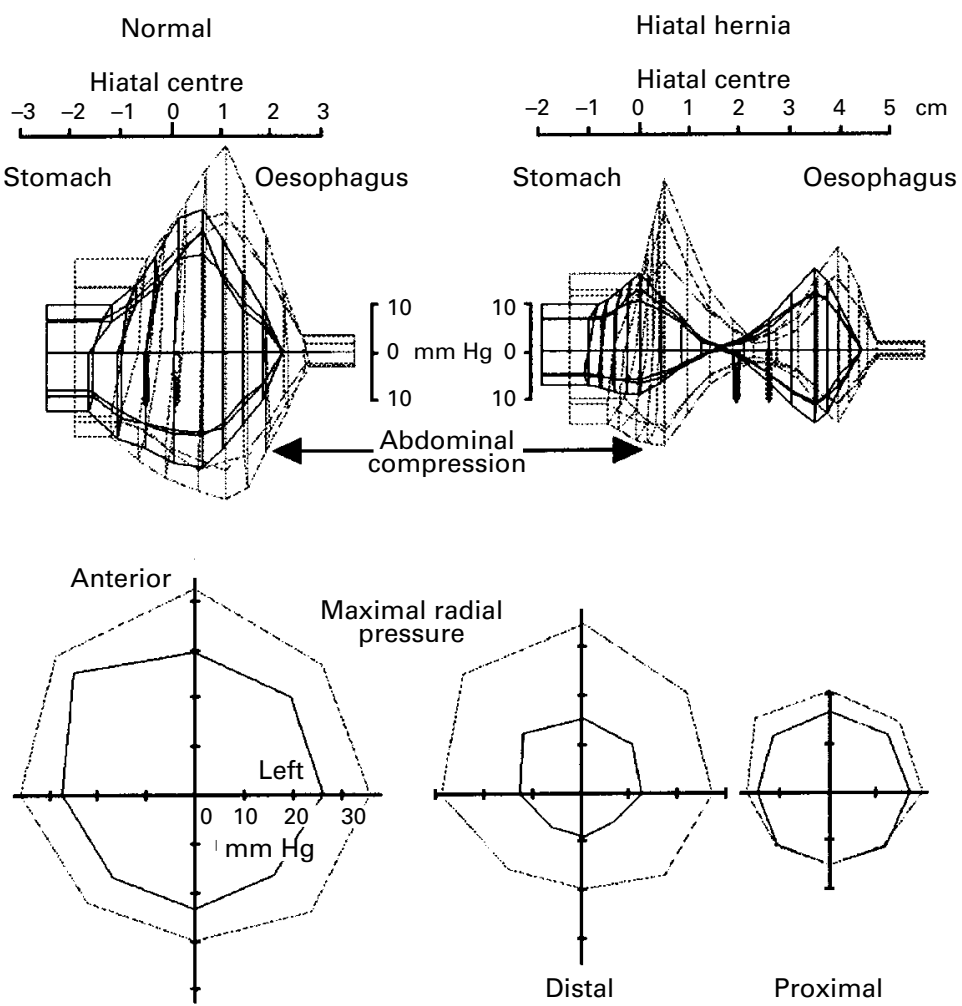

Figure 4 Change in the pressure topography of the gastro-oesophageal junction resulting from abdominal compression with an abdominal binder. The solid lines represent the condition at rest (represented as in fig 3) and the broken lines show movement and pressure augmentation associated with abdominal compression. Note that abdominal compression shifts the position of the squamocolumnar junction proximally by about $0.5 \mathrm{~cm}$, increases the intragastric pressure, and causes a substantial increase in pressure within the hiatal canal of the hernia subjects. All values of length and pressure are the medians of the subject groups.

nine axial positions in the case of a single high pressure zone and 17 in the case of a double peaked axial pressure profile. Group data for normals and hiatal hernia subjects were summarised by the median axial coordinate (relative to position zero) and the corresponding median pressure value of each axial position of each peak of each radial side hole orientation of each pull through condition. Three dimensional representations of gastrooesophageal junction pressure topography relative to the hiatus and mucosal clips were created from the median data for each group and condition by aligning the radial pressure profiles relative to each other using a computer workstation (Silicon Graphics Personal IRIS;

Table 3 Changes in pressure, length, and position of the gastro-oesophageal junction high pressure zone caused by Müller manoeuvre

\begin{tabular}{llll}
\hline & \multicolumn{3}{l}{ Hiatus hernia } \\
\cline { 3 - 4 } & $\begin{array}{l}\text { Normal } \\
\text { subjects }\end{array}$ & $\begin{array}{l}\text { HPZ 1 } \\
\text { (proximal) }\end{array}$ & $\begin{array}{l}\text { HPZ } 2 \\
\text { (hiatal) }\end{array}$ \\
\hline Intragastric pressure change (mm Hg) & $10(2)$ & - & $8(1)$ \\
Distal oesophageal pressure change (mm Hg) & $-5(2.0)$ & $-5(1.5)$ & - \\
Increase in maximal pressure (mm Hg) & $27(1.3)$ & $-3(0.2)^{\star}$ & $24(1.4)$ \\
Length change of high pressure zone (cm) & $-0.8(0.2)$ & $0.4(0.1)^{\star}$ & $0.3(0.1)^{\star}$ \\
Movement of locus of maximal pressure (cm) & $-2.2(0.5)$ & $-0.6(0.2)^{\star} \dagger$ & $-1.8(0.4)$ \\
Movement of squamocolumnar junction (cm) & $-1.1(0.3)$ & $-0.4(0.2)^{\star}$ & - \\
Movement of hiatal centre (cm) & $-1.7(0.4)$ & - & $-1.8(0.4)$ \\
\hline
\end{tabular}

Changes in the hiatus hernia patients were measured both within the proximal high pressure zone (HPZ 1) and the hiatal canal (HPZ 2).

All values are mean $(\mathrm{SEM})$

${ }^{\star} \mathrm{p}<0.05$ versus normal; $\mathrm{t} \mathrm{p}<0.05$ versus movement of $\mathrm{HPZ} 2$.
Silicon Graphics, Mountain View, California, USA) and three dimensional graphics and animation software (Advanced Visualizer, Version 4.1, Wavefront Technologies, Santa Barbara, California, USA).

Calculations of percentage radial asymmetry for pressure peaks identified by the methodology in fig 2 were made by applying the formula: percentage asymmetry $=(1-$ minimal radial pressure/maximal radial pressure $) \times$ 100. Thus, a circle has $0 \%$ asymmetry. Comparisons of pressure topography characteristics between groups and conditions were made using Student's paired or unpaired $t$ test.

Simulation of hiatal hernia reduction was done mathematically by repositioning the squamocolumnar junction of the hiatal hernia subjects (guided by the mucosal clip) along with the associated sphincter pressures to the median subdiaphragmatic position observed in the normals. The modelled radial pressure values at each axial coordinate of the simulated hernia reduction thus equalled the algebraic sum of pressure attributable to the stationary distal high pressure zone and corresponding repositioned proximal high pressure zone values.

\section{Results}

PRESSURE TOPOGRAPHY OF THE

GASTRO-OESOPHAGEAL JUNCTION

Median pressure values at axial and radial coordinates of the gastro-oesophageal junction high pressure zones of normal and hiatal hernia subjects are diagrammed as three dimensional wireframes in fig 3 . In each case, the median position of the squamocolumnar junction is indicated by the proximal mucosal clip while the median position of the intragastric margin of the gastro-oesophageal junction is indicated by the distal mucosal clip. Table 1 shows corresponding pressure, length, and radial asymmetry characteristics of the high pressure zones. A distinguishing feature of the hernia group was a double peaked pressure profile. As evidenced by the maximal radial pressure profiles in the lower panels of fig 3, the distal high pressure zone had notable radial asymmetry while the proximal one was nearly circular. In the hernia subjects, the centre of the distal high pressure zone occurred $2.1(1.0) \mathrm{cm}$ distal to the squamocolumnar junction, corresponding precisely with the anatomical location of the hiatal canal as determined by prior fluoroscopic analysis. In the normal subjects, however, the median position of the squamocolumnar junction was 0.5 $(0.1) \mathrm{cm}$ distal to the centre of the hiatal canal and the intragastric margin of the high pressure zone extended $1.1(0.1) \mathrm{cm}$ distal to the squamocolumnar junction. These spatial relations imply that the distal high pressure zone of the hernia subjects was attributable to the extrinsic effect of the hiatal canal (as opposed to the intrinsic oesophageal sphincter) as normal subjects exhibited no high pressure zone at a location so distal to the squamocolumnar junction. 

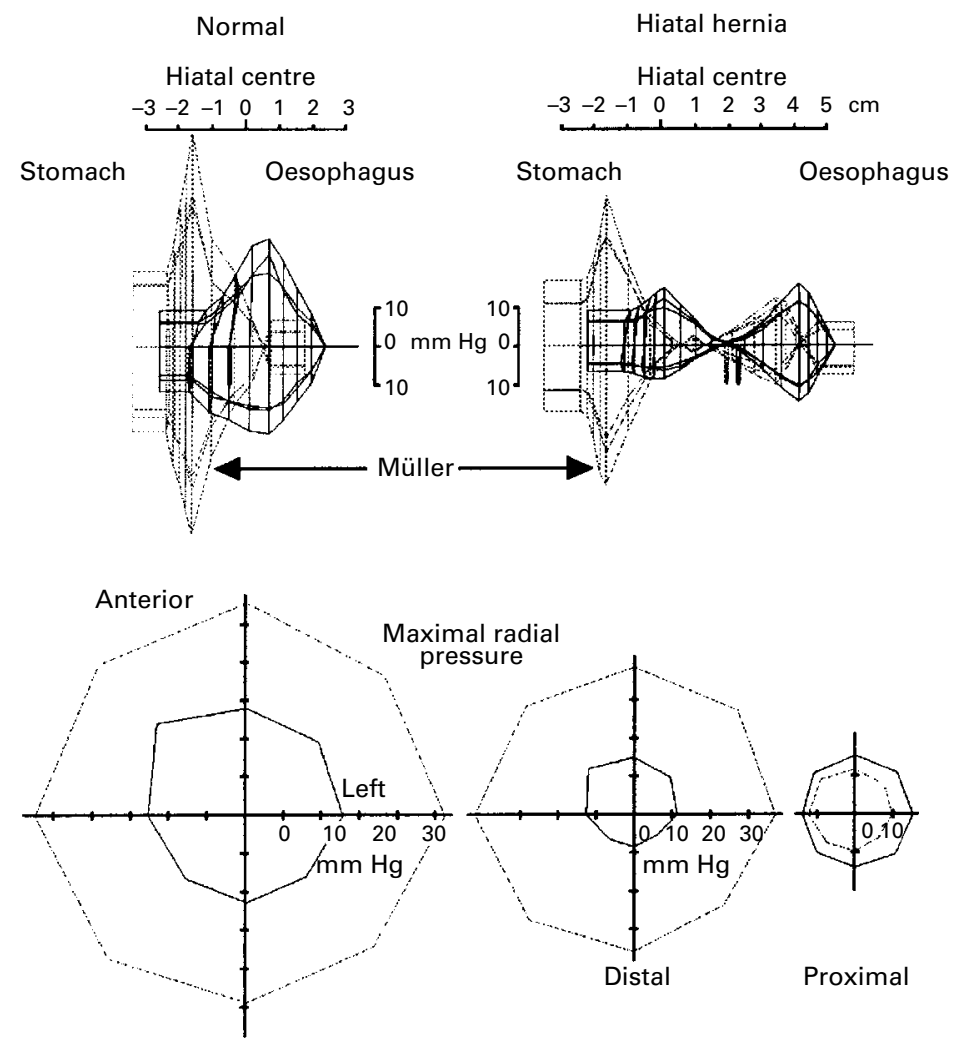

Figure 5 Change in the pressure topography of the gastro-oesophageal junction resulting from a Müller manoeuvre. The solid lines represent the condition at rest (represented as in fig 3) and the broken lines show movement and pressure augmentation associated with inspiration against a closed glottis. Note that the Müller manoeuvre shifts the position of the squamocolumnar junction distally by more than $1 \mathrm{~cm}$ in the normals but by less than 0.5 $\mathrm{cm}$ in the patients with hiatus hernia. In both subject groups, the Müller manoeuvre increases the intragastric pressure, and causes a substantial increase in pressure within the hiatal canal. All values of length and pressure are the medians of the subject groups.

DYNAMIC EFFECTS OF ABDOMINAL COMPRESSION AND THE MÜLLER MANOEUVRE

Abdominal compression augmented the gastro-oesophageal junction pressure of both the normal subjects and the patients with hiatal hernia as evident in fig 4 . Abdominal compression also resulted in proximal shifting of both the hiatal centre and squamocolumnar junction relative to the vertebral reference point by approximately $0.5 \mathrm{~cm}$ in all cases, with no apparent discordance between the degree of diaphragmatic movement and squamocolumnar junction movement (table 2). Within the hernia group, abdominal compression augmented the pressure within the distal (hiatal) element to a degree that was equal to the pressure augmentation observed in normal

Table 4 Comparison of parameters of the gastro-oesophageal junction high pressure zone and its response to abdominal compression (AC) and Müller manoeuvre of normal subjects, patients with hiatus hernia, and patients with a hernia after algebraic simulation of hiatus hernia reduction

\begin{tabular}{llll}
\hline & $\begin{array}{l}\text { Normal } \\
\text { subjects }\end{array}$ & $\begin{array}{l}\text { Hiatus } \\
\text { hernia }\end{array}$ & $\begin{array}{l}\text { Simulated } \\
\text { reduction }\end{array}$ \\
\hline Maximal pressure (mm Hg) & $28(1.2)$ & $17(1)^{\star}$ & $27(1.6)$ \\
Axial length (cm) & $4.0(0.2)$ & $5.8(0.4)^{\star}$ & $2.9(0.1)^{\star}$ \\
Length proximal to SCJ (cm) & $2.8(0.2)$ & $2.6(0.4)$ & $2.6(0.3)$ \\
Length distal to SCJ (cm) & $1.1(0.1)$ & $2.9(0.1)^{\star}$ & $0.5(0.1)^{\star}$ \\
Percentage radial asymmetry & $34(2)$ & $52(2)^{\star}$ & $38(2)$ \\
Maximal pressure with AC (mm Hg) & $41(1.2)$ & $26(1.5)^{\star}$ & $44(1.1)$ \\
Maximal pressure with Müller manoeuvre (mm Hg) & $55(1.3)$ & $37(1.4)^{\star}$ & $55(1.6)$ \\
\hline
\end{tabular}

All pressures are referenced to atmospheric pressure. SCJ, squamocolumnar junction. All values are mean $(\mathrm{SEM})$.

${ }^{\star} \mathrm{p}<0.05$ versus normal. subjects (table 2). Although augmentation of the proximal element of the high pressure zone was also evident in the patients with hiatus hernia, this was to a lesser degree than that of the hiatal element. Furthermore, the radial asymmetry of the proximal pressure augmentation, with anterior pressure dominating, was similar to the radial pressure asymmetry within the hiatus (fig 4).

The Müller manoeuvre augmented the gastro-oesophageal junction high pressure zone and shifted both the hiatal centre and the high pressure zone distally in the normal subjects (fig 5, top left). In those with hiatal hernia, however, although the distal element of the high pressure zone was augmented to a similar degree to that seen among the normal subjects (fig 5, table 3), the magnitude of the proximal element of the high pressure zone was slightly reduced as was the pressure in the more proximal oesophagus $(-5 \mathrm{~mm} \mathrm{Hg})$. Figure 5 and table 3 also show that movement of the proximal and distal high pressure zones in the patients with hiatal hernia during the Müller manoeuvre was unequal. While the distal high pressure zone shifted $1.8 \mathrm{~cm}$ distally with the Müller manoeuvre, consistent with that observed in the normal subjects, the proximal element moved only a third as much. Thus, while the normal subjects exhibit a 1.1:1.7 ratio of movement between the squamocolumnar junction and diaphragm, this ratio is reduced to $0.4: 1.8$ in those with hiatus hernia, thereby permitting more movement of the diaphragm relative to the oesophagus.

SIMULATION OF HERNIA REDUCTION

Using the fluoroscopically ascertained position of the hiatal centre, and maintaining a fixed relation between the proximal high pressure zone and the endoscopically marked squamocolumnar junction, hiatal hernia reduction was simulated by repositioning the proximal high pressure zone a measured distance (mean 2.6 $\mathrm{cm}$ ) into the hiatal canal. Figure 6 illustrates the resultant gastro-oesophageal junction pressure topography. As table 4 shows, this manipulation transforms the high pressure zone of the patients with hiatal hernia into one with many similarities to that of the normal subjects; in particular, maximal pressure, percentage asymmetry, and dynamic response to both abdominal compression and the Müller manoeuvre. The only significant difference between the pressure topography of the normal subjects and patients with hiatal hernia after the simulation of hernia reduction was in the overall length of the gastro-oesophageal junction high pressure zone. When this remaining difference is subdivided into segments proximal and distal to the squamocolumnar junction, it is evident that the difference results primarily from characteristics distal to the squamocolumnar junction (fig 6 (shaded areas), table 4).

\section{Discussion}

A high pressure zone detectable by intraluminal oesophageal manometry and attributable to the extrinsic effect of the diaphragmatic hiatus 


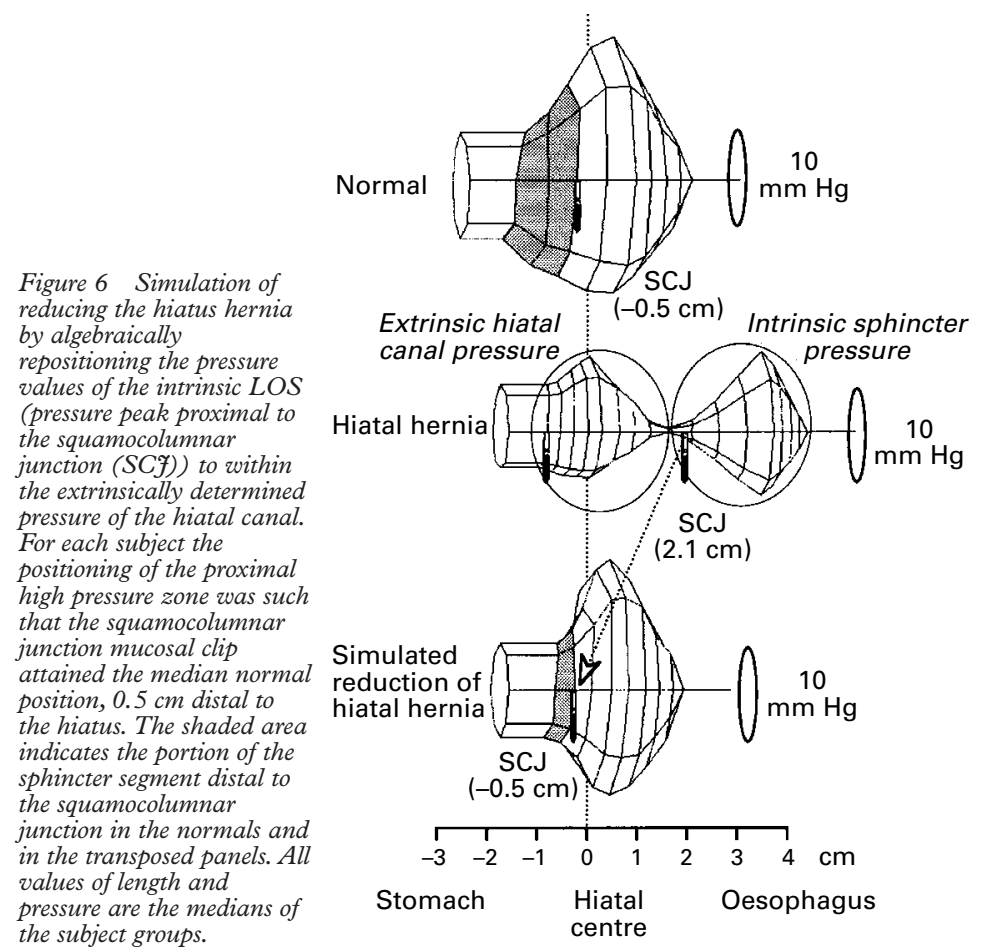

was initially described in the opossum, a species characterised by anatomic separation of the LOS and hiatus. ${ }^{16}$ In that work, Schulze et al noted this effect as the likely explanation for the double pressure peak observed in pull through recordings obtained in some patients with hiatus hernia as described by Code et al. ${ }^{17}$ In the present investigation, we used similar methodology to define the pressure topography of the gastro-oesophageal junction relative to the squamocolumnar junction and to the diaphragmatic hiatus in subjects with and without sliding hiatus hernia. Endoscopically placed clips were used to localise the squamocolumnar junction relative to radio-opaque markings on a manometric assembly as the assembly was withdrawn across the gastrooesophageal junction during fluoroscopy. Findings in the patients with hiatus hernia showed that, even in end expiration, the hiatal canal contributed to the overall pressure topography of the gastro-oesophageal junction as evident by a double peaked pressure profile. Assuming that extrinsic and intrinsic pressures are additive when superimposed, this separation of the intrinsic LOS from the hiatal canal results in an obligate decrement of peak gastro-oesophageal junction pressure with hiatus hernia. Conversely, utilising the metal clip at the squamocolumnar junction as a landmark to gauge the appropriate degree of reduction of the hernia, repositioning the intrinsic lower oesophageal sphincter back within the hiatal canal effectively restored a normal maximal pressure. Furthermore, our findings suggest that the normal radial asymmetry of the gastro-oesophageal junction pressure is attributable to the extrinsic contribution of the hiatal canal, as the intrinsic LOS was radially symmetric in the patients with hiatus hernia.
The observation that the hiatal canal contributes to gastro-oesophageal junction pressure in patients with hiatus hernia during both inspiration and expiration is consistent with previous observations made in both humans and animals. Klein et al studied the thoracoabdominal junction of 10 patients after surgical resection of the lower oesophageal sphincter as part of cancer therapy. ${ }^{18}$ Subsequent manometric analysis revealed an end expiratory intraluminal pressure of 6 (SE 1) $\mathrm{mm} \mathrm{Hg}$ within the "sphincterless" gastro-oesophageal junction, a value very similar to the $3(0.2) \mathrm{mm} \mathrm{Hg}$ observed within the hiatal canal of our patients with hiatus hernia. Relevant animal data come from experimentally severing the phrenooesophageal ligament in dogs; analogous to the effect of axial hiatus hernia in which the ligament is stretched and its diaphragmatic attachments loosened. ${ }^{19} 20$ Severing the ligament substantially reduced peak gastrooesophageal junction pressure, which was then restored with reanastomosis. ${ }^{21}$ The only contradictory data seem to be from diaphragmatic electromyography (EMG) recordings which strongly support the notion of a phasic, but not tonic, diaphragmatic contribution to gastrooesophageal junction pressure. ${ }^{12}{ }^{13}$ However, relying on EMG recordings to represent completely the diaphragmatic contribution to gastro-oesophageal junction pressure ignores the possible contribution of passive forces such as diaphragmatic and ligamentous elasticity to intraluminal pressure. Certainly, in the case of the upper oesophageal sphincter, such passive forces contribute an intraluminal pressure of similar magnitude after experimental abolition of the myogenic tone. ${ }^{22}$

Patients with hiatus hernia exhibited augmentation of gastro-oesophageal junction pressure of similar magnitude to normal subjects in response to either abdominal compression or deep inspiration (figs 4 and 5; table 2). Pressure augmentation in the patients with hiatus hernia was almost entirely attributable to the diaphragmatic sphincter component; the minimal (asymmetric) changes observed proximal to the squamocolumnar junction were probably attributable to overlap with the diaphragmatic segment. This hypothesis is supported by the brisk diaphragmatic EMG responses to these sphincteric challenges described both in humans and animals. The magnitude of the resultant gastro-oesophageal junction pressure augmentation paralleled that of the integrated EMG signal and was unaffected by concomitant intrinsic sphincter relaxation induced by oesophageal balloon distension or swallowing. ${ }^{23-26}$ These observations, coupled with the observation that hiatus hernia progressively compromises the competence of the gastro-oesophageal junction to increases in intra-abdominal pressure, ${ }^{11}$ suggest that axial separation of the diaphragmatic sphincter component from the intrinsic sphincter is detrimental to its function. A possible mechanism for this functional decrement is entrapment of gastric juice above the hiatus, a phenomenon exacerbated by loosening of phrenooesophageal attachments which allows sub- 
stantial movement of the intrinsic sphincter element relative to the hiatus (table 2).

The most confusing segment of oesophageal anatomy is that distal to the squamocolumnar junction, referred to by Inglefinger as an anatomic and functional "no mans land". ${ }^{27}$ Highlighting this confusion, Wolf remarked that, "it is indeed strange that, when normally located below the hiatus, the 'submerged segment' resembles the esophagus while, when displaced above the hiatus, it resembles stomach. In fact, when a large hiatal hernia is present, the original submerged segment is incorporated into the hernia sac". ${ }^{28}$ Our measurements suggest that when intra-abdominal length is defined as the segment of the high pressure zone distal to the centre of the hiatal canal, this is mainly a determination of whether or not hiatus hernia is present (fig 3). In normal subjects, this extends $1.1 \mathrm{~cm}$ distal to the squamocolumnar junction and $1.6 \mathrm{~cm}$ distal to the hiatal canal (fig 6). Anatomical studies by Liebermann-Meffert et al support this estimate of the extent that the sphincteric segment extends distal to the squamocolumnar junction. ${ }^{29}$ These authors described a "fold transition line", evident in postmortem specimens which seems analogous to the intragastric margin of the gastro-oesophageal junction as we defined it endoscopically. LiebermannMeffert et al describe this as the area of greatest muscle wall thickness, oriented obliquely, and related to the angle of His as identified externally. The squamocolumnar junction was 10.5 (SD 4.4) $\mathrm{mm}$ proximal to the fold transition line when measured along the greater curvature, almost identical to the 11 (SE 1) $\mathrm{mm}$ calculated using our methodology. Although the relevance of this distal sphincter segment is controversial, Hill et al found the integrity of this "flap valve" to correlate with gastrooesophageal junction competence against an antegrade pressure gradient in postmortem experiments. ${ }^{14}$ The intriguing observation that this is the one aspect of gastro-oesophageal junction pressure topography not restored by simulated reduction of a hiatal hernia tends to support the widely held surgical opinion that effective antireflux surgery must additionally entail some reconstruction of this segment. ${ }^{1430}$

In summary, this experiment correlated anatomical, manometric, and radiographic representations of the gastro-oesophageal junction in normal subjects and in individuals with hiatus hernia. The major findings of the work suggest that hiatus hernia alters the pressure topography of the gastro-oesophageal junction by reducing its maximal pressure, altering its radial asymmetry, and reducing the sub-hiatal length of the high pressure zone. All of these changes, except the latter, can be reversed by simulating reduction of hiatus hernia. The implication of these findings is that by altering the pressure topography of the gastrooesophageal junction, hiatus hernia may increase susceptibility to reflux events associated with diminished LOS pressure-for example, strain induced or free reflux, as opposed to reflux by transient LOS relaxation.
This work was supported by grant RO1 DC00646 (PJK) from the Public Health Service.

1 Dent J, Dodds WJ, Friedman RH, et al. Mechanism of gastroesophageal reflux in recumbent asymptomatic human troesophageal reflux in recumbent asy
subjects. $\mathcal{F}$ Clin Invest 1980;65:256-67.

2 Dodds WJ, Dent J, Hogan WJ, et al. Mechanisms of gastroesophageal reflux in patients with reflux esophagitis. $N$ Engl f Med 1982;307:1547-52.

3 Kahrilas PJ, Dodds WJ, Hogan WJ, et al. Peristaltic dysfunction in peptic esophagitis. Gastroenterology 1986;91:897904.

4 Csendes A, Maluenda F, Braghetto I, et al. Location of the lower esophageal sphincter and the squamous columnar mucosal junction in 109 healthy controls and 778 patients with different degrees of endoscopic oesophagitis. Gut with different

5 Cadiot G, Bruhat A, Rigaud, et al. Multivariate analysis of pathophysiological factors in reflux oesophagitis. Gut 1997;40:167-74.

6 Barham CP, Gotley DC, Alderson D. Precipitating causes of acid reflux episodes in ambulant patients with gastrooesophageal reflux disease. Gut 1995;36:505-10.

7 Dent J, Holloway RH, Toouli J, et al. Mechanisms of lower oesophageal sphincter incompetence in patients with symptomatic gastrooesophageal reflux. Gut 1988;29:10208 .

8 Petersen KH, Myrvold HE, Grette K, et al. Hiatus hernia in gastroesophageal reflux disease. Scand $\mathcal{f}$ Gastroenterol 1986;21:31-4

9 Sontag SJ, Schnell TG, Miller TQ, et al. The importance of hiatal hernia in reflux esophagitis compared with lower esophageal sphincter pressure or smoking. F Clin Gastroenterol 1991;13:628-43.

10 Weberg AR, Larsen F, Hoel B, et al. Relationship of hiatus hernia to reflux oesophagitis. Scand $\mathcal{F}$ Gastroenterol 1986;21:55-8.

11 Sloan S, Rademaker AW, Kahrilas PJ. Determinants of gastroesophageal junction incompetence: hiatus hernia, lower esophageal

12 Boyle JT, Altschuler SM, Nixon TE, et al. Role of the diaphragm in the genesis of lower esophageal sphincter pressure in the cat. Gastroenterology 1985;88:723-30.

13 Mittal RK, Rochester DF, McCallum RW. Electrical and mechanical activity in the human lower esophageal sphincter during diaphragmatic contraction. $f$ Clin Invest 1988;81:1182-9.

14 Hill LD, Kozarek RA, Kraemer SJM, et al. The gastroesophageal flap valve: in vitro and in vivo observations. Gastrointest Endosc 1996;44:541-7.

15 Logemann JA, Kahrilas PJ, Begelman J, et al. Interactive computer program for biomechanical analysis of videoradiographic studies of swallowing. AfR Am 7 Roentgenol 1989;153:277-80

16 Schulze K, Dodds WJ, Christensen J, et al. Esophageal manometry in the opossum. Am F Physiol 1977;233:E152-9.

17 Code CF, Kelley ML, Schlegel JF, et al. Detection of hiatal hernia during esophageal motility tests. Gastroenterology 1962;43:521-31.

18 Klein WA, Parkman HP, Dempsey DT, et al. Sphincterlike thoracoabdominal high pressure zone after esophagogastrectomy. Gastroenterology 1993;105:1362-9.

19 Kahrilas PJ, Wu S, Lin S, et al. Attenuation of esophageal shortening during peristalsis with hiatus hernia. Gastroenterology 1995;109:1818-25.

20 Friedland GW. Historical review of the changing concepts of lower esophageal anatomy: 430BC-1977. AfR Am f Roentgenol 1978;131:373-88.

21 Michelson E, Siegel CI. The role of the phrenico-esophageal ligament in the lower esophageal sphincter. Surg Gyn Obstet 1964;118:1291-4.

22 Asoh R, Goyal RK. Manometry and electromyography of the upper esophageal sphincter in the opossum. Gastroenterology 1978;74:514-20.

23 Boyle JT, Altschuler SM, Nixon TE, et al. Responses of feline gastroesophageal junction to changes in abdominal feline gastroesophageal junction to changes
pressure. Am f Physiol 1987;253:G315-22.

24 Mittal RK, Rochester DF, McCallum RW. Sphincteric action of the diaphragm during a relaxed lower esophageal sphincter in humans. Am F Physiol 1989;256:G139-44.

25 Mittal RK, Fisher M, McCallum RW, et al. Human lower esophageal sphincter pressure response to increased intra-abdominal pressure. Am f Physiol 1990;258:G62430 .

26 Martin CJ, Dodds WJ, Liem HH, et al. Diaphragmatic contribution to gastroesophageal competence and reflux in dogs. Am f Physiol 1992:263: G551-7.

27 Ingelfinger FJ. Esophageal motility. Physiol Rev 1958;38: 533-84.

28 Wolf BS. Sliding hiatal hernia: the need for redefinition. $A F R$ Am F Roentgenol 1973;117:231-47.

29 Liebermann-Meffert D, Allgöwer M, Schmid P, et al. Muscular equivalent of the lower esophageal sphincter. Gastroenterology 1979;76:31-9.

30 Stein HJ, DeMeester TR, Naspetti R, et al. Threedimensional imaging of the lower esophageal sphincter in gastroesophageal reflux disease. Ann Surg 1991;214:37484 . 